\title{
Core-Shell Nanofibers: Nano Channel and Capsule by Coaxial Electrospinning
}

\author{
Fengyu $\mathrm{Li}^{1,2}$, Yong Zhao ${ }^{1}$ and Yanlin Song ${ }^{1}$ \\ ${ }^{1}$ Institute of Chemistry Chinese Academy of Sciences, Beijing, \\ ${ }^{2}$ Bowling Green State University, Bowling Green, $\mathrm{OH}$, \\ 1China \\ 2USA
}

\section{Introduction}

In the last 30 years, nano science and technology have an unprecedented development. Nanofibers, one-dimensional (1D) nanostructures have been paid great attentions due to their unique properties and intriguing applications in many areas (Xia et al., 2003). In lots of synthetic and fabrication methods, electrospinning (a drawing process based on electrostatic interactions) provided the simplest approach to nanofibers with exceptionally long in length, uniform in shape, and diversified in composition (Li et al., 2004; Greiner et al., 2007). Following the fast and polymer common property, scientists invented complex electrospinning. Utilizing electrostatic interactions and through the elongation of viscoelastic jet device, electrospinning technique can copy the structure of jet device to nanoscale fibers from polymer solution or molten. Various nanofibers with different secondary nanostructure were fabricated. Especially, core-shell nanofibers pull more and more interesting and attention, because of the great potential applications and prospects for nanochannel, nanocapsule and small encapsulating devices. Coaxial electrospinning is an effective, fast and controlled technique to construct core-shell nanostructures into nanofibers (Li et al, 2004; Zhang et al., 2004 and Xia et al., 2006).

Here, based on complex electrospinning technique, we will describe nanofibers with nanochannel and core-shell nano-capsule fabrication, and how coaxial electrospinning is working to construct various nanostructures in the nanofibers. Furthermore, we will introduce some encapsulation of stimulating responsive materials into core-shell nanofibers, and the improvement of the responsibility and functional devices applications for the combination of core-shell nanofibers and stimulating responsive materials.

\section{Coaxial electrospinning}

Electrospinning, also known as electrostatic spinning, has been developed since 1990s as micro-nano fiber and particle fabrication technique (Reneker et al, 1996; Huang et al, 2003). It uses an electrical charge to draw a very fine (typically on the micro or nano scale) fibers from liquid. Figure 1 shows the common setup and working principle of electrospinning. A high voltage and a cuspidate spinneret provided by a point electrode creates a charge aggregation into the polymer solution or liquid at the spinneret of syringe. Under pulling of 
electrostatic force, polymer liquid is drawn and got a "Taylor cone" at the jut of spinneret, then is drawn out from spinneret as the "Liquid jet" part in Figure 1. Due to the electrostatic repulsion of the charges in the polymer liquid, the polymer liquid is drawn thinner and thinner, as the fiber spin part in Figure 1. In this process, the ultrathin fibers solidify or dry rapidly, and then the nanofibers are collected on the "Collector".

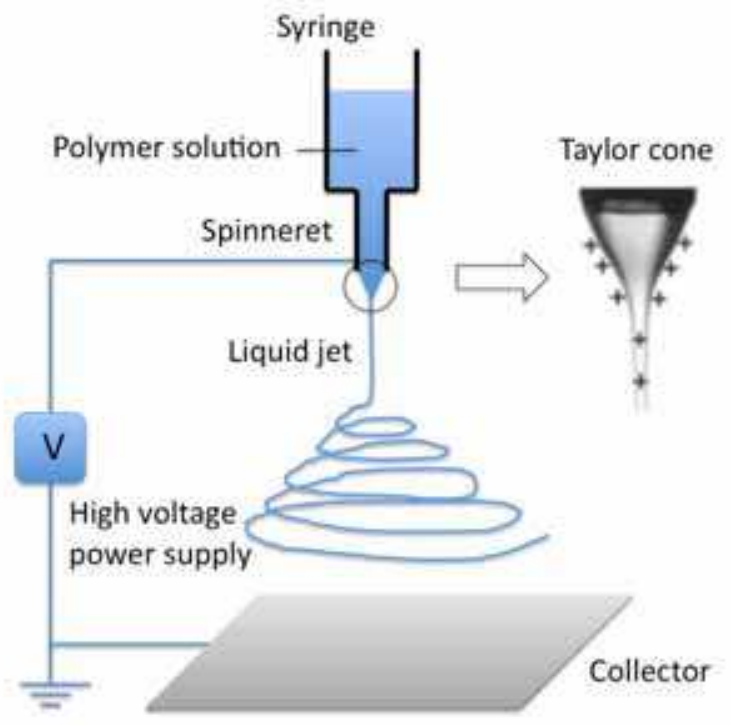

Fig. 1. The common setup and working principle of electrospinning.

Electrospinning shares characteristics of both electrospraying and conventional solution dry spinning of fibers. The spinning fibers process is non-invasive and does not require the use of coagulation invasion to produce solid threads from liquid. It makes the process particularly suitable for the production of fibres using macromolecule or polymer. In theory, electrospinning can be applied to all polymer to get nanofibers.

Nanofibers which be prepared by electrospinning, usually exhibit a solid interior and smooth surface. If an appropriate modification is employed to the electrospinning, it can prepare nanofibers with some specific secondary structure. Complex electrospinning was developed from basic electrospinning to fabricate nanofibers with secondary structures. Here we only discuss the core-shell nanofibers and the preparation method: "Coaxial electrospinning".

As shown in Figure 1, the process of fiber spinning after "Liquid jet", it is non-invasive, just thinner and thinner. It means, the "Taylor cone" plays an important role in the moulding of nanofibers. One can make a compound spinneret to modify "Taylor cone" to prepare nanofibers with complex secondary structures.

Figure 2 shows a basic setup for coaxial electrospinning and the fabrication process of common core-shell nanofibers. Based on the basic electrospinning setup, two syringes feed inter-separated and coaxial "Inner fluid" and "Outer fluid" to spinneret. Under high voltage, the electrospinning liquid is drawn out from spinneret and forms a "Compound Taylor cone" with a core-shell structure (Loscertales, et al. 2002). After "Coaxial jet", the 
core-shell structure will be built and kept in the fibers through spinning solid and be collected on the "Collector". In those processes, core-shell Taylor cone formation will decide the core-shell nanofibers forming and fabrication. In order to get a nice "Compound Taylor cone" with a core-shell structure, one has to make an utmost control and balance inject speed of inner and outer fluid, which keep the "Compound Taylor cone" in dynamic stabilization. A too high or too low speed of inner fluid is unfavourable. An appropriate injecting speed and rate of inner-outer fluid should be considered and investigated.

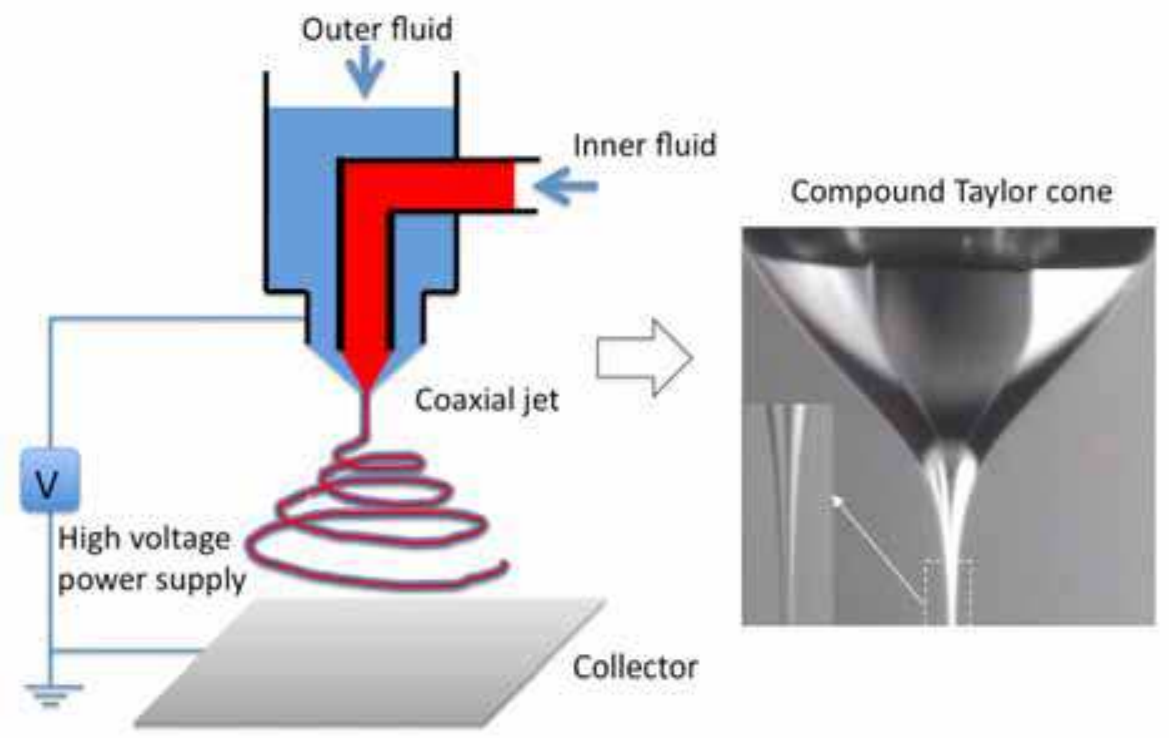

Fig. 2. The basic setup for coaxial electrospinning and fabrication process of common coreshell nanofibers.

Many scientists gave the abundant and excellent contribution on hollow and core-shell nanofibers by coaxial electrospinning after 2000s' respectively (Loscertales et al, 2002; Su et al, 2003; Li et al, 2004; Zhang, et al., 2004; Li et al, 2009). They fabricated hollow nanofibers with polyvinylpyrrolidone (PVP) and polycaprolactone (PCL) polymer system respectively. Figure 3 shows the hollow nanotube structure constructed by Prof. Xia's group. In order to prevent mixing between inner and outer fluid in Taylor cone, the mineral oil mostly was used as the inner fluid.

In the compound-jet electrospinning process, the outer polymer solution and inner paraffin oil were co-issued from respective nozzles, the outer liquid flowed through the gaps between the capillary and the formed liquid jacket enveloped the inner fluid. When the high voltage was applied, the conductive outer solution was charged and the compound liquid was stretched and whipped to a thin liquid thread in order to release the static electric repulsion by dispersing electric charge (Doshi et al, 1995). However, this repulse force could not act on inner liquids directly because of the insulation of paraffin oil, which means that the paraffin oil cannot be electrospun on its own. As a result, the inner fluids were subjected to the pressure transferred from the outer fluid and were compressed to thin liquid thread 
accompanied with outer liquid. At the same time, with the evaporation of solvent and gelation of polymer, the outer liquid shell solidified very quickly which suppressed the Reyleigh instability of the whipping jet. Consequently, solid tubes with independent channel were formed in which paraffin oil was enveloped.

After coaxial electrospinning, the fibers with a polymer shell and oily core were produced first. By removal of organics through calcinations, a $\mathrm{TiO}_{2}$ hollow tube was obtained. In Figure 3, the walls of these tubes were made of a composite containing amorphous $\mathrm{TiO}_{2}$ and $\mathrm{PVP}$. $\mathrm{TiO}_{2}$ (anatase) hollow fibers were obtained by calcining the composite nanotubes in air at $500{ }^{\circ} \mathrm{C}$. SEM image of a uniaxially aligned array of anatase hollow fibers that were collected across the gap between a pair of electrodes. These fibers were fractured using a razor blade to expose their cross-sections. In the preparation of all these samples, the feeding rate for heavy mineral oil was $0.1 \mathrm{~mL} / \mathrm{h}$ and the concentrations of $\mathrm{Ti}(\mathrm{OiPr})_{4}$ and $\mathrm{PVP}$ were 0.3 and $0.03 \mathrm{~g} / \mathrm{mL}$, respectively. The voltage of electrospinning was $12 \mathrm{kV}$. Those fibers show an uniform, circular cross-section long and hollow nanostructure. They are well-separated from each other and can be conveniently transferred onto other substrates for a variety of applications. The circular cross-sections and well-controlled orientation of the hollow nanofibers should make them particularly useful as nanofluidic channels. The procedure can be extended to many other materials with potential applications in catalysis, sensing, encapsulation, and drug delivery.

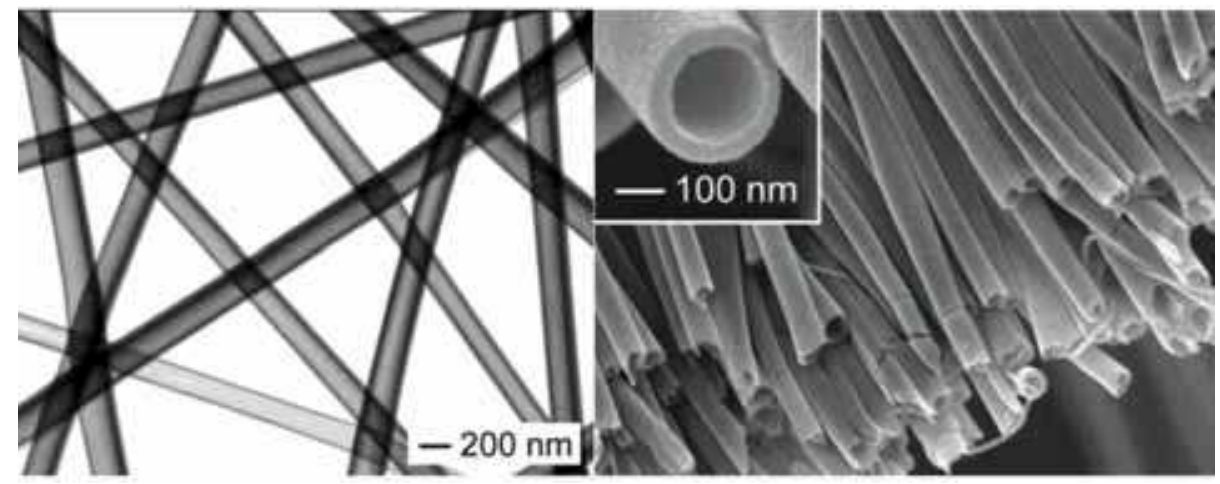

Fig. 3. TEM image (left) and SEM image (right) of $\mathrm{TiO}_{2} / \mathrm{PVP}$ hollow fibers fabricated by coaxial electrospinning.

\section{Multi-channel coaxial electrospinning and Nano-channel nanofibers}

\subsection{Multi-channel coaxial electrospinning}

One-dimensional nanomaterials with hollow interiors have considerable applications in micro/nanofluidic devices, drug delivery, catalysis and others (Hu et al, 1999; Law et al, 2004). Coaxial electrospinning showed promising method for building ultralong nanotubes. However, most of the nanotubes are possess only one single inner channel. As the aspect and prospects of nano and functional materials research and development currently and future, the multifunctional, integrative and miniature devices research is urgently expected. Dr. Zhao and Prof. Jiang designed and carried out the strategies of building nanomaterials with more complex inner structures. Here we will describe a novel multi-fluidic compoundjet electrospinning technique in detail, which could fabricate hierachical multi-channel nanotubes in an effective way. 




Fig. 4. Schematic illustration of multi-channel coaxial electrospinning system.

The experimental setup of multi-fluidic compound-jet electrospinnig is sketched in Figure 4. Several metallic capillaries with outer diameter (O.D.) of $0.4 \mathrm{~mm}$ and inner diameter (I.D.) of $0.2 \mathrm{~mm}$ were arranged at the several vertexes of an equilateral triangle. Then the bundle of capillaries was inserted into a plastic syringe (O.D. $=3.5 \mathrm{~mm}, \mathrm{I} . \mathrm{D} .=2.0 \mathrm{~mm}$ ) with gaps between individual inner capillaries and outer syringe. Two immiscible viscous liquids were fed separately to the three inner capillaries and outer syringe in an appropriate flow rate. A 20\% Polyvinylpyrrolidone (PVP) ethanol solution, served as outer liquid, while a nondissolution paraffin oil was chosen as inner liquid. Then a high voltage generator between three inner metallic capillaries and a metallic plate coated with a piece of aluminum foil acted as counter electrode provide the driving and controlling for the electrospinning. The immiscible inner and outer fluids (red for paraffin oil and blue for $\mathrm{Ti}(\mathrm{OiPr})_{4}$ solution) were issued out separately from individual capillaries. With a suitable high voltage application, a whipping compound fluid jet is formed under the spinneret and then a fibrous membrane is collected on the aluminium foil.

A rational design of spinneret is utmost important for the successful fabrication of multichannel fibers by coaxial electrospinning. The inject speed of inner and outer fluid must provide an elaborate control and balance. Further more, the gaps between each capillary and the capillary to the inner wall of outer syringe are equal, to ensure each inner capillary is isolated from other two capillaries and the outer nozzle. The outer fluid should surround the inner fluids effectively, and the inner liquids should flow out independently and don't mix with each other.

Because of surface tension, liquid column should prefer shrinking to cylinder to acquire the smallest surface area. But, the insection of inner channels of mulit-channel fibers is flabellate rather than circular, which represent the shape of inner liquids. Figure 5 shows the 


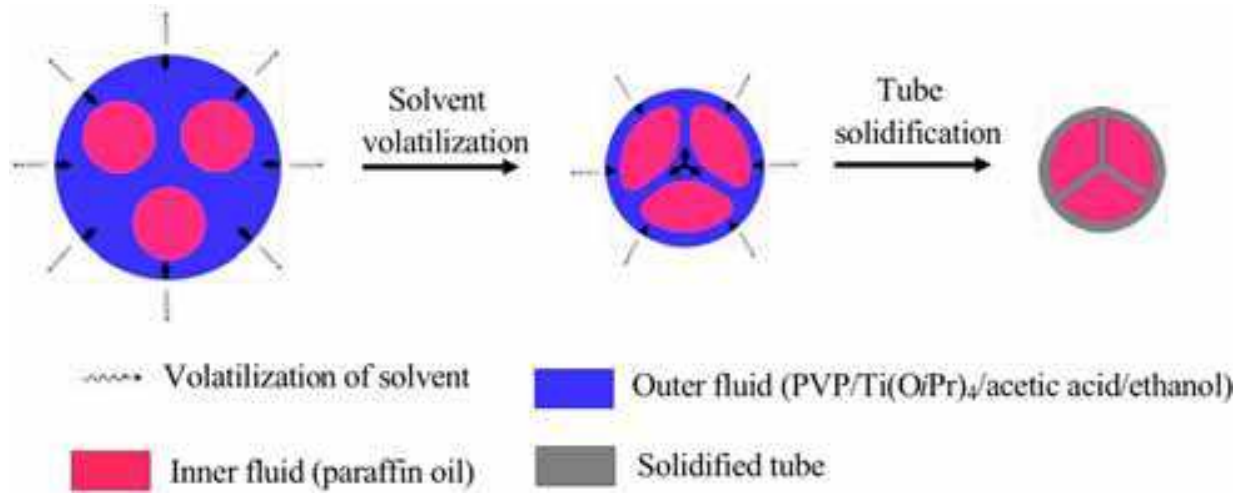

Fig. 5. Schematic illustration of multi-channel fiber shaping.

proposed formation process for multi-channel tube. (Here, a three-channel tube served as an example.) Two immiscible liquids with different properties are in the multi-fluidic compound electrospinning system. The outer liquid is a multi-component solution, in which the content of volatile solvent is in the majority, while the inner paraffin oil is a nonvolatile liquid. In the initial stage of electrospinning process that the compound fluid jet just leaves the spinneret, both outer and inner fluids are of cylinder shape according to the morphology of spinneret. With bending and whipping of the liquid jet in air, the volume of outer fluid shrinks remarkably by the lose of solvent. Then a dilemma emerge that either outer fluid or inner fluids must deform under the shrink pressure because the shrunk tube shell cannot hold the three cylinder inner fluids any more. Thermodynamic analysis indicates that the deformation of inner fluids is favorable for the stability of system. The interfacial tension of the outer and inner liquids is much smaller than outer liquid the surface tension of outer liquid (Adamson et al, 1997). The deformation of outer solution needs more energy than that of inner liquids. To lower the total free energy of the compound fluid, the outer fluid is drained to surface through the liquid node and border film (called Plateau border) between three inner fluids by capillary suction. When the Plateau border suction is counterbalanced by the disjoining pressure then reaches a mechanical equilibrium state, the border film between neighbouring inner fluid becomes flat (Exerowa et al, 1998; Höhler et al, 2005). With the drainage of outer fluid, the inner liquids evolve to three flabellate liquid columns. Plateau's law indicates that three angles between Plateau border are equal $\left(120^{\circ}\right)$ under thermodynamic equilibrium conditions, which agree well with the mutual angle of Y-shape inner ridge of three-channel nanotube. Generally speaking, to lower the free energy of the compound fluid system, the inner fluids transform to flabellate shape under the shrinkage pressure of outer liquid and form multi-channel nanotube with multi-pointed star shape inner ridge ultimately. The multi-fluidic compound-jet electrospinning technique breaks through the limit at two fluids system that could generate programmable multi-channel or multi-component $1 \mathrm{D}$ nanomaterials in an effective way.

\subsection{Mulit-channel nanofibers}

After an utmost control compound-jet electrospinning process and follow-up treatments (the inner channels of the tube correspond to the vacancy of the inner fluids after they were removed), Figure 6 and Figure 7 show the SEM images of the multi-channel fibers prepared by coaxial electrospinning. The fibers have uniform, flat and smooth surface. The side-view 
was checked to expose the cross sections of the multi-channel fibers. It can be clearly seen that most of tubes are of hollow structures with multi-cavum, circular and closed outer wall of the hollow tube. The diameter distribution of the tubes is relative uniform with average value of $2.3 \mu \mathrm{m}$ (in a sub-nano size). The inner diameter of the channels is around 100-500 $\mathrm{nm}$ size. Decades nanometer walls made the compartment of the several cavums. The through cavums form the nano-channels in the polymer or inorganic fibers and give the huge surface area. The multi-furcate ridge embeds in the outer tube and exhibits an interesting mulit-pointed star " $Y$ " or " $X$ " shape, and the ridges partition the nanotubes into several flabellate parts. Like a scaffold, the multi-furcate ridges support the hollow structure, and make the hollow structure have higher intensity.
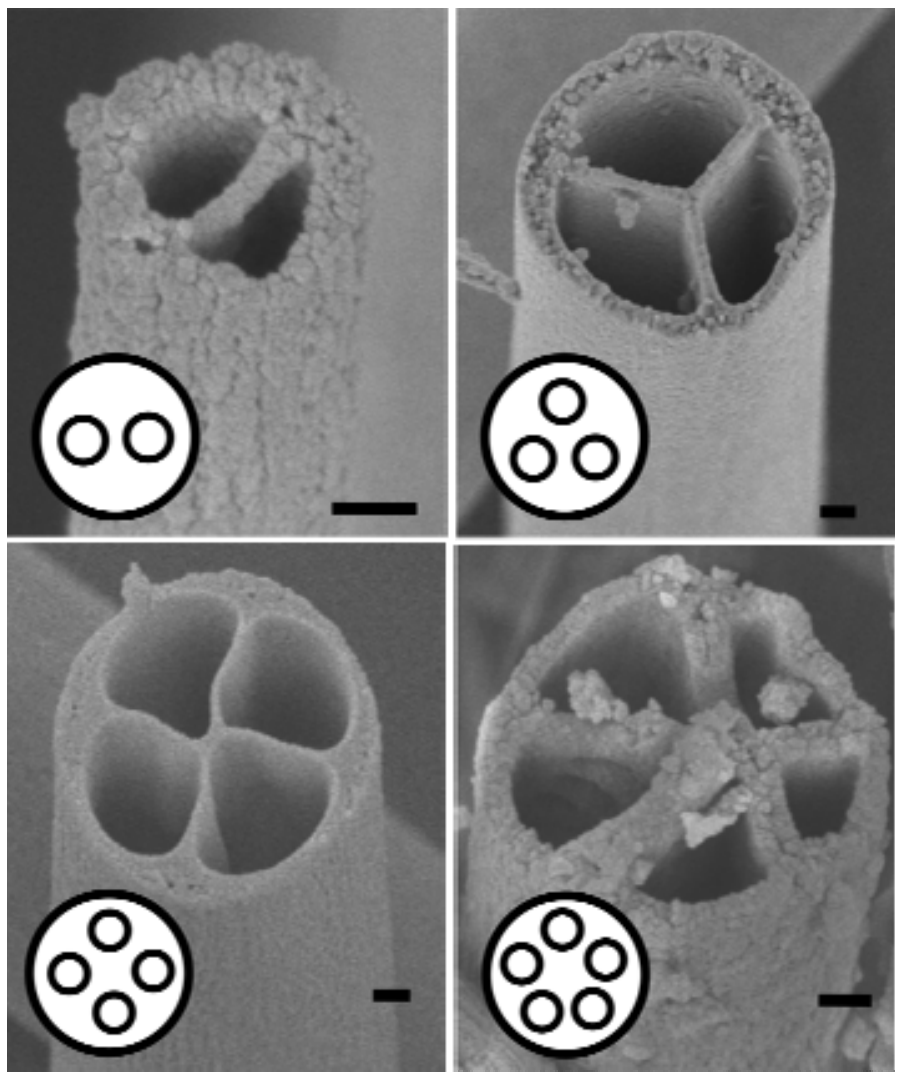

Fig. 6. The cross section SEM images of hollow fibers with two, three, four and five channels. The scale bars are $100 \mathrm{~nm}$.

In Figure 6, nanotubes with two, three, four and five channels have been successfully fabricated by multi-channel coaxial electrospinning. All of the multi-channel nanofibers show good fidelity to the corresponding spinneret. With different inner fluid speed control of multi-fluidic compound-jet, one would fabricate the two or three channel nanofibers with different inner diameters. That reveals the efficient controllability to construct multi-channel tube with different shapes in polymer nanofibers. The multi-channel coaxial electrospinning 
has good diversity. It demonstrates that multi-channel coaxial electrospinning could fabricate all kinds of multi-channel nanofiber in theory.
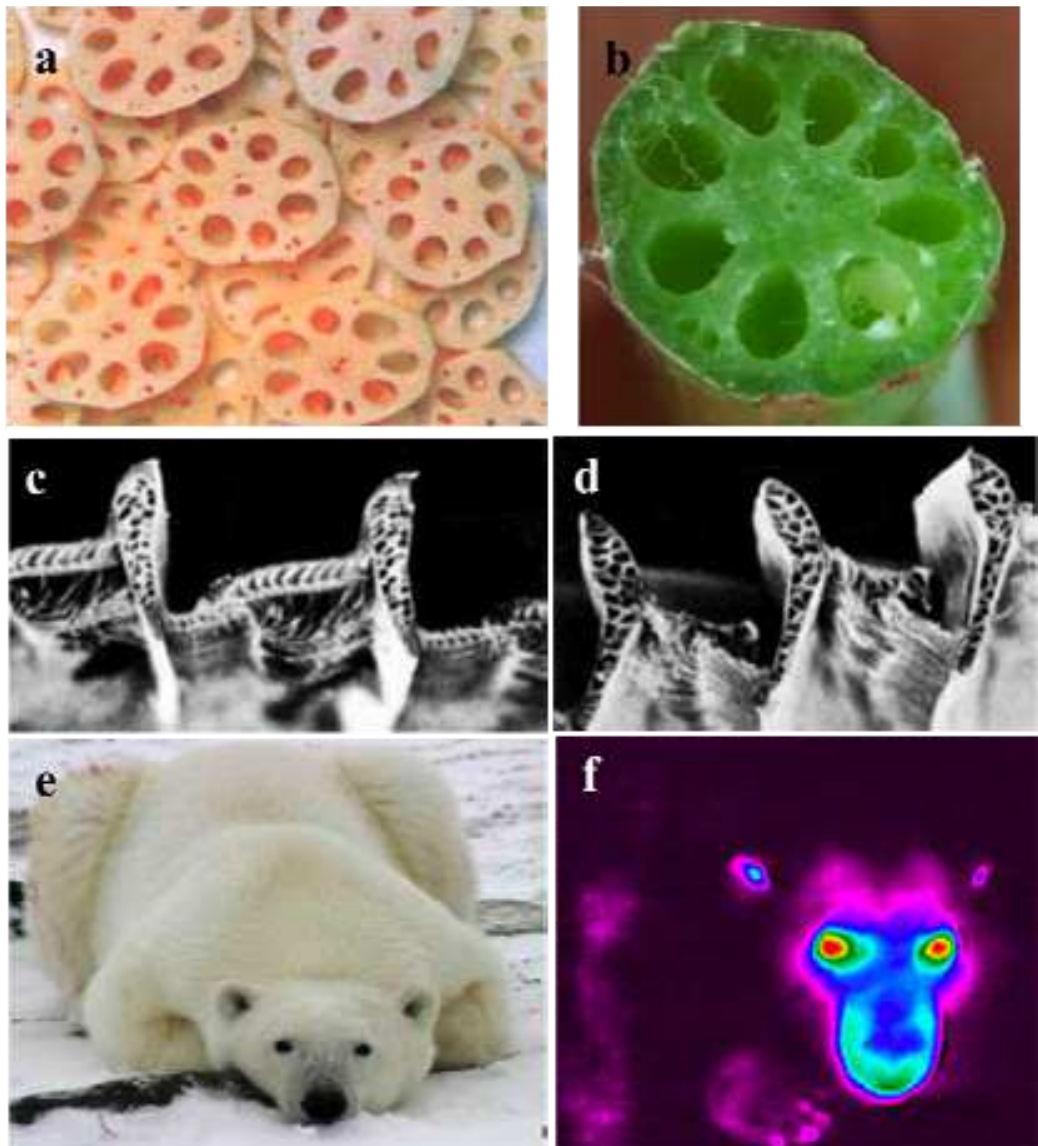

f
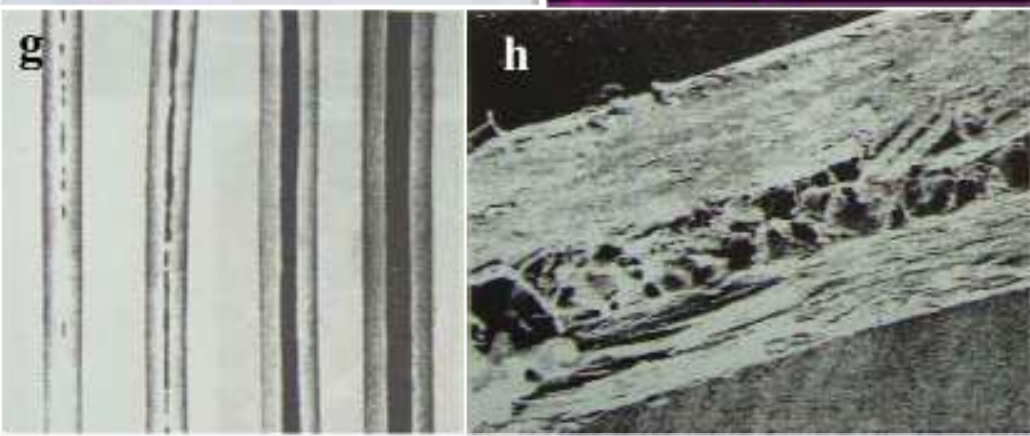

Fig. 7. Schematic of the multichannel structure in biology, which show great means of multichannel fibers in bionics. Materials delivery multichannel in lotus root a)-b), and multicavum structure for anti-cold in aves feather c)-d), and polar bear hair e)-h). 
In application and biology, multichannel tubes or fibers have great importance and prospect. Figure 7 displays several typical examples of the multichannel structure in biology. There are multichannel in lotus root for the materials delivery. Multicavum structure in aves feather makes the feather ultra-light and high intensity. And multicavum structure in polar bear hair makes it have prefect temperature keeping and anti-cold property. (The infrared image of polar bear in Figure $7 \mathrm{f}$ shows that the heat energy losing only happens on the eyes, nose and ears, where are no or less hair covered parts.) High similarity with biological micro- or nanostructures and large areas, fast fabrication will give multi-channel fibers and multi-channel coaxial electrospinning wide prospects for research and application.

Compared with single channel, multi-channel structures may possess considerable advantages such as independent addressable channels, better mechanical stability, unique thermal properties and larger surface-to-volume area. Furthermore, by replacing inner fluids with other functional molecular, multicore-shell nanofibers can be created and different components can be integrated in nanodomain without interaction. Such nanofibers would have novel and improved properties that do not exist in each component. They might be promising candidates for a wide range of applications such as bionic super lightweight, thermo-insulated textiles, high efficiency catalysis, vessels for macro/nano fluidic devices in bioscience or lab-on-a-chip and multi-component drug delivery. This general method could be readily expanded to many other materials.

\section{Melt coaxial electrospinning and Nano-encapsulation and capsule in nanofibers.}

Multiplicity, controllability and applicability are the aspect and prospects of nano-science and nano-technology. At the same time for improving multiplicity and controllability, materials scientists pay great attention on the application of the new nano-technology or the new application of general nano-technology. Following diversification development of responsive materials and nano-technology, the combination of various functional materials with nano structures are drawing much attention for the great prospect in smart materials and devices, which always can generate new materials with prominent functions (Gil et al, 2004; Lu et al, 2007). The core-shell nanomaterials give small capsule to encapsulate the responsive or functional materials. And the coaxial electrospinning technique is an easy and fast process to build kinds of core-shell nanofibers.

In the fore part of this chapter, we have discussed coaxial electrospinning can perform good control, and fabricate core-shell and hollow nanofibers fast and simply. In the coaxial electrospinning process of fabricating polymer nanofibers, the inner fluid and outer fluid should be delaminated and without mutual mixing, for example water and dichloromethane (DCM), to keep a clear interface between core and shell of the nanofibers. However, for good conductivity and solubility, most good solvents for electrospinning are amphiphilic, for example dimethylformamide (DMF), dimethyl sulfoxide (DMSO), tetrahydrofuran (THF) and ethanol. But as a fast process of electrospinning, if the outer fluid polymer solution was dry and the inner fluid was solidify before the mixture, the nice coreshell structure still can be bullied in the polymer nanofibers.

\subsection{Melt coaxial electrospinning}

In 2006, based on coaxial electrospinning, Prof. Xia's group made the first try and invented melt coaxial electrospinning to fabricated phase change materials encapsulation core-shell 
nanofibers (McCann et al., 2006). They appended a heating system on the conventional coaxial electrospinning setup to provide a thermal atmosphere for the fluidic inner fluid. The melt coaxial electrospinning experimental setup is shown in Figure 8. The heating tape with a temperature controller device on the inner fluid syringe was used to keep the inner fluid molten and fluid. Two syringe pumps were used to perform the utmost control of the inner fluid (melt hydrocarbon phase change materials) and outer fluid ( $\mathrm{PVP} / \mathrm{Ti}(\mathrm{OiPr})_{4}$ solution) respectively. The two fluids met at the metallic needle spinneret, which was built to coaxial cannula. Electrospinning relied on the use of a high-voltage electric field to draw a viscous droplet into an elongated jet. Under high-voltage service, the liquid was pulled out from spinneret to fibers thinner and thinner. In this process, the inner melt hydrocarbon phase change materials fluid froze rapidly, and the outer fluid polymer solution dried and solidified fast.

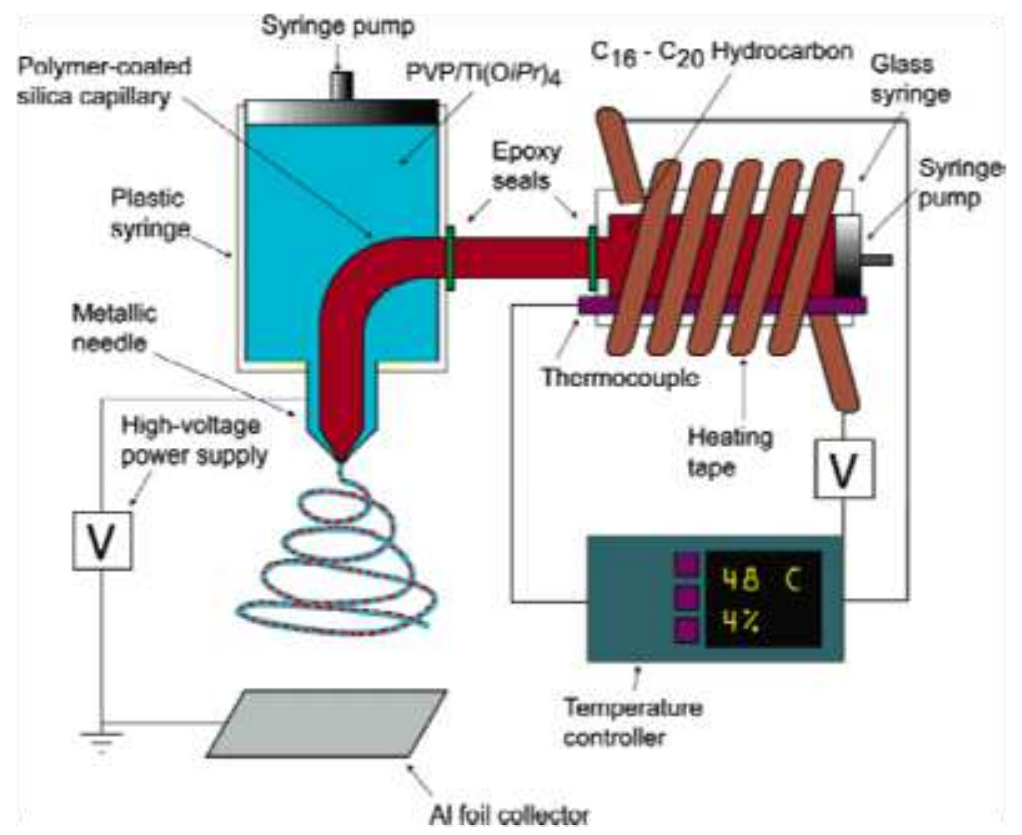

Fig. 8. Schematic of the melt coaxial electrospinning setup used for fabricating $\mathrm{TiO}_{2}-\mathrm{PVP}$ nanofibers loaded with hydrocarbon PCMs.

2009, Dr. Li and Prof. Song made an improvement for the melt coaxial electrospinning (Li et al, 2009). The melt coaxial electrospinning experimental setup is shown in Figure 9. In addition to the high voltage generator supply and the syringe pump controller, a whole thermal atmosphere heating system was build into the conventional coaxial electrospinning setup. Two injectors with different diameter and needles constructed the outer and inner dopes loading setup. In practice, the whole thermal atmosphere of loaded system was proved more propitiously to prevent the inner dope's freeze by jamming of the needle. (For low phase change temperature materials, an infrared lamp will be easier to supply a whole thermal atmosphere for the loaded system.) Keeping the inner inject materials (the phase change materials) fluid before it is spun out from spinneret should be treated with an 
utmost care and control. It is the key factor to keep the propitious encapsulation of phase change materials into the inner of the fibers and get the Phase Change Materials (PCMs)Polymer core-shell fibers with a high yield filling.

A dynamic instability resulted in whipping and stretching which was responsible for the attenuation of the jet into long fibers with ultrathin diameters. Electrospinning was remarkably simple and versatile and capable of producing nano- and microscale fibers in large quantities. Polymer solutions were predominantly used in this process, though composites, sol-gels, and surfactant-based solutions had also been included to fabricate nanofibers with a broad range of compositions, morphologies, and properties.

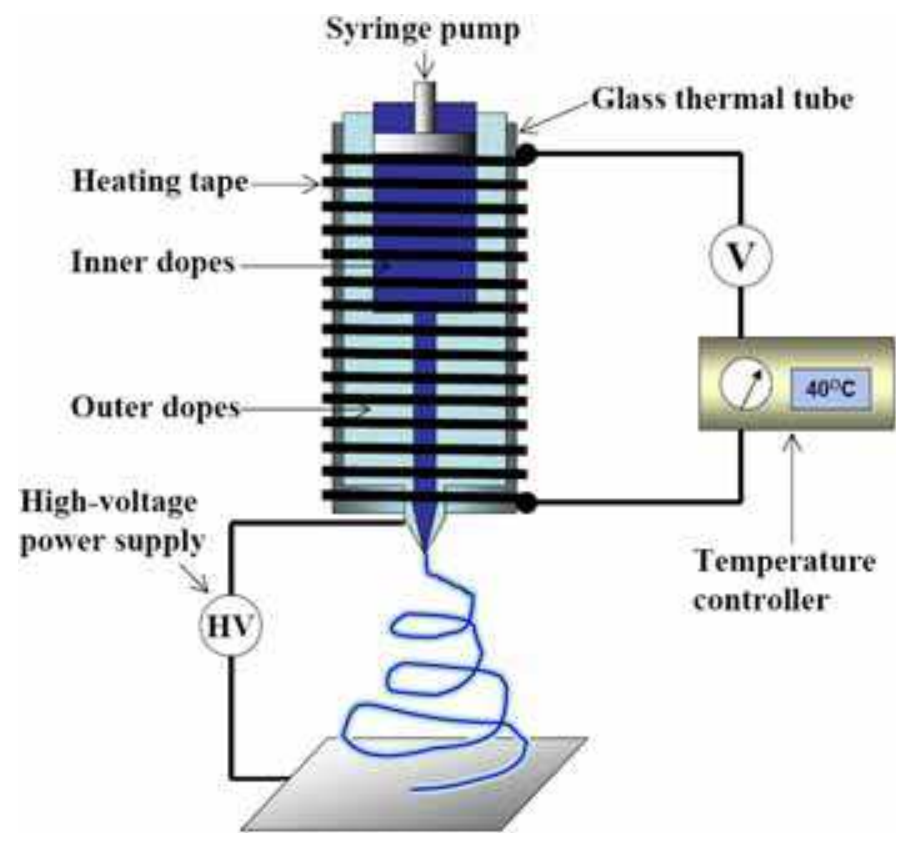

Fig. 9. The melt coaxial electrospining setup with a whole thermal atmosphere for the loaded system used for fabricating polymer nanofibers loaded with phase change materials.

\subsection{Nano-encapsulation and capsule in nanofibers.}

\subsubsection{Phase change materials encapsulation in nanofibers.}

Scanning electron microscopy (SEM) images and transmission electron microscopy (TEM) images can clearly recal the secondary nanostructures of the fibers electrospun with a melt coaxial spinneret. Figure 11 shows the Octadecane@ $\mathrm{TiO}_{2}-\mathrm{PVP}$ nanofibers electrospun with a melt coaxial spinneret by Prof. Xia's setup (Figure 8). The sheath consisted of a $\mathrm{TiO}_{2}-\mathrm{PVP}$ composite while the core was octadecane. The core material was heated up to melt temperature for injection. In Figure 10 (A) and (C) show the SEM images of the as-prepared fibers with different hydrocarbon materials loading, and (B) and (D) show the corresponding TEM images of the fibers after they have been soaked in hexane for $24 \mathrm{~h}$ to remove the hydrocarbon core. Those fibers were 100-200 $\mathrm{nm}$ in average diameter. The TEM images indicate that the octadecane formed spherical droplets or elongated, compartmentalized domains along the long axis of the fiber. 

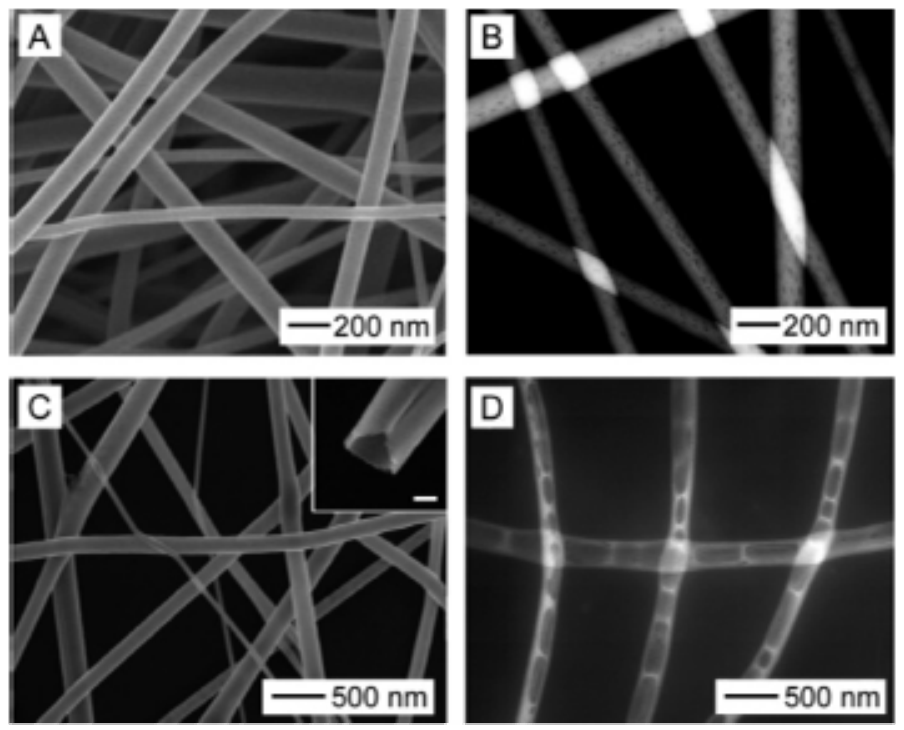

Fig. 10. Octadecane@ $\mathrm{TiO}_{2}-\mathrm{PVP}$ nanofibers electrospun with a melt coaxial spinneret. SEM images and TEM images of the nanofibers with 7\% (A)-(B) and 45\% (C)-(D) octadecane loading.

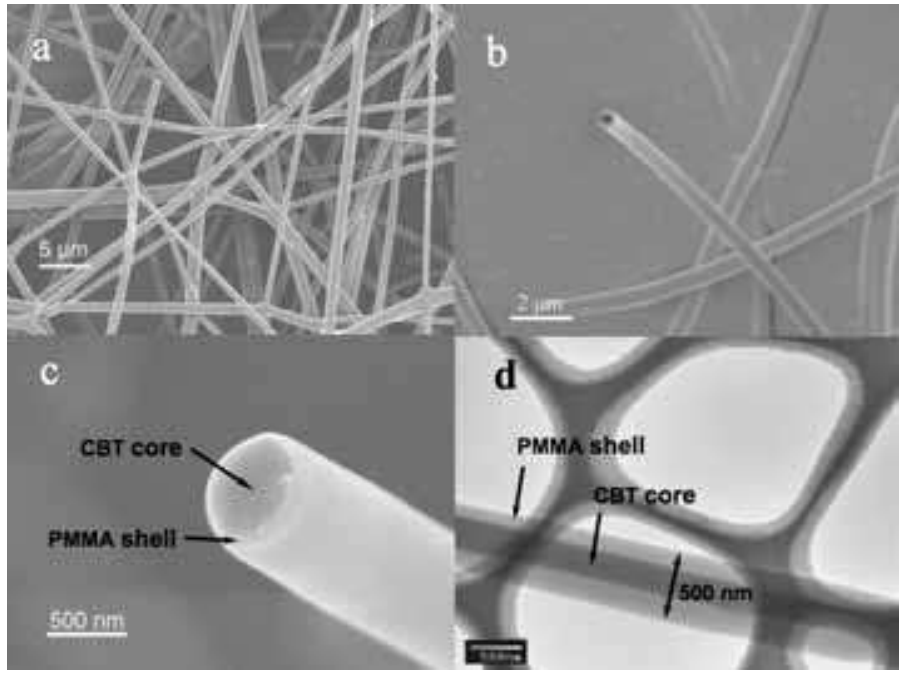

Fig. 11. Tetradecanol@PMMA nanofibers electrospun with a melt coaxial spinneret. SEM images of nanofibers in wide area (a) and side-view of core-shell nano- fiber lateral sections (b)-(c), and core-shell structure in TEM images (d).

Figure 11 is the Tetradecanol@PMMA nanofibers electrospun with a melt coaxial spinneret by Prof. Song's setup (Figure 9), which shows the actual loading state of core-shell nanofibers. (No core-removing) The sheath consisted of optical transmission polymer 
ploy(methyl methacrylate) (PMMA) while the core was 1-tetradecanol and phase transformation developer composite (CBT). Figure 11a indicates the SEM image of the fibers with smooth surface and $500 \mathrm{~nm}$ to $2 \mu \mathrm{m}$ average diameter. In the side-view SEM image of Figure $11 b$ and 11c, core-shell structure of nanofiber lateral sections was displayed. TEM image in Figure 11d reveals the clear interface between 1-tetradecanol core and PMMA polymer shell, and $200 \mathrm{~nm}$ inner diameter and $500 \mathrm{~nm}$ outer diameter. It is the actual core-shell nano-fibers. Further more, it indicates that the core-material was encapsulated independently and phase separated from polymer matrix shell wall. That will be the most important fact to keep the thermo-responsive, energy-storage and management properties of the phasetransformation.

\subsubsection{Stimulation chromic materials encapsulation in nanofibers.}

As a kind of classic responsive material, phase change materials (PCMs) were attracted much attention for the phase transformation absolutely reversibility and good energy storage and management property. (Muligan, et al., 1996; Zalba, et al, 2003) However, the fluidity of the phase change materials after melting made the PCMs hard to fix and stabilize, which limited the practical application. It is necessary to stabilize PCMs in a solid matrix. The melt coaxial electrospining just gives a suitable and ideal process and method to encapsulate and stabilize the PCMs into the nanomatrix of core-shell polymer fibers. The core-shell structure gives the free space out of the polymer matrix for their phase change. As illustrated in Figure 12, the PCMs were encapsulated and stabilized in the centre cavum, where it could perform the melt and crystallization independently and no interruption. And with the nano-encapsulation, the fluidity of the melt PCMs will be utmost limited by the strong capillarity of the nanotubes. While huge surface of nanofibers provide huge heat area when the temperature change, which should make a more sensitive thermo-responsive property of the PCMs. It provides the new insight into the preparation of temperature sensors, calefactive materials with energy absorption, retention, and release.
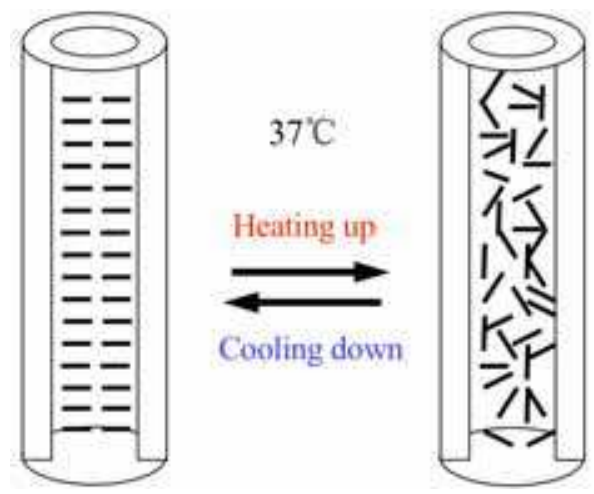

Fig. 12. Reversible phase transformation process in core-shell nanofiber of phase change materials.

Figure 13 displays the experiment for the capability of these PCM Octadecane@ $\mathrm{TiO}_{2}-\mathrm{PVP}$ nanofibers to stabilize temperature. A borosilicate glass vial was covered with a different insulation jacket, filled with quantitative $60{ }^{\circ} \mathrm{C}$ water and then allowed to cool in a $4{ }^{\circ} \mathrm{C}$ environment. The water temperature in the vial was measured using a thermal couple every 
$30 \mathrm{~s}$ and recorded until it reached $20^{\circ} \mathrm{C}$. Curve A was no insulation jacket on the glass vial; curve $B$ and $C$ was half and whole insulated with a $2 \mathrm{~mm}$ thick layer of octadecane@ $\mathrm{TiO}_{2}-$ PVP fibers sandwiched between Al foils on the vial; and curve D was the vial covered with an $8 \mathrm{~mm}$ thick jacket of fiberglass fibers. Supercooling of vial A was not observed in the temperature history curve for the PCM nanofibers. And the fiberglass fibers were as effective as the PCM nanofibers in insulating the vial with a 4 times thicker cover. The PCM fibers cover had a temperature stabilization time (close to the melting point of octadecane) for $5 \mathrm{~min}$. It gave an evident energy release in the cooling process, which indicated a practical applicable energy storage and management character.

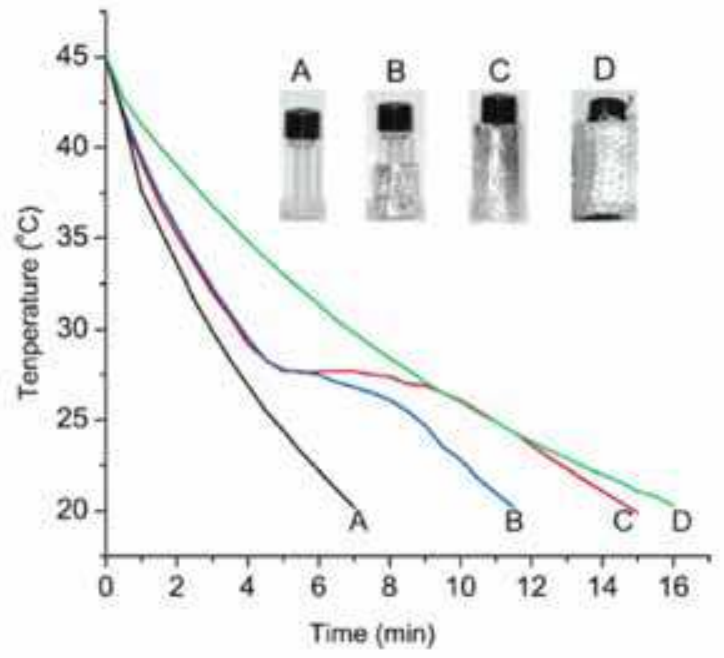

Fig. 13. Demonstration of thermal insulation capability of octadecane@TiO2-PVP nanofibers, where $1 \mathrm{~cm}^{3}$ of water at $60^{\circ} \mathrm{C}$ was allowed to cool in a $4{ }^{\circ} \mathrm{C}$ environment in glass vials covered with different insulation jackets.

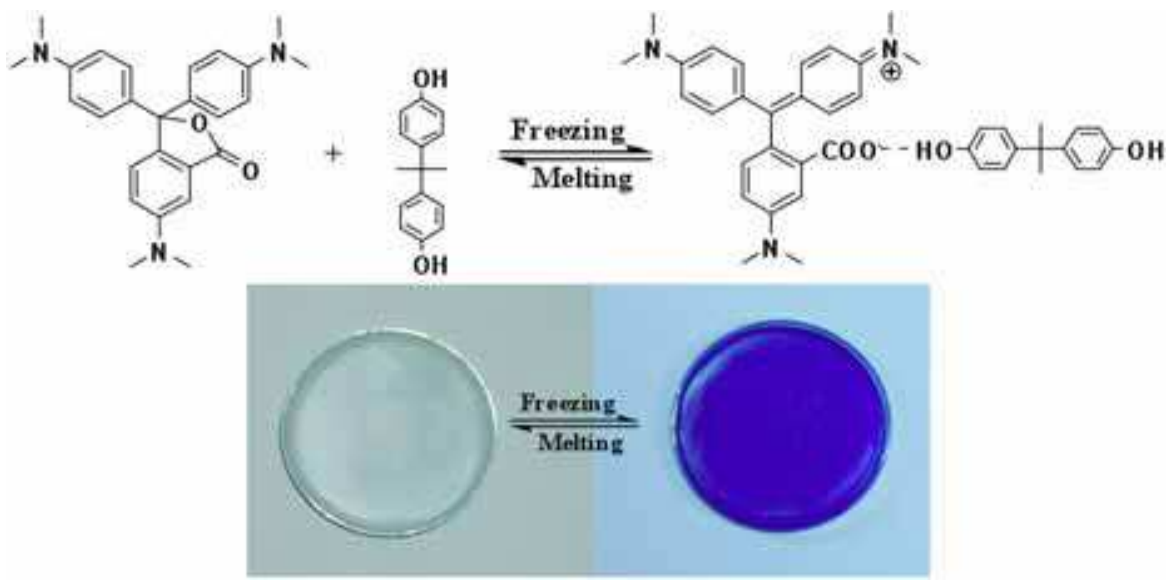

Fig. 14. Schematic of phase change thermochromic material CVL, bisphenol Ae and 1tetradecanol (CBT) thermochromism in the phase-change process. 
To realize more application, Dr. Li and Prof. Song introduced the thermochrom into the PCMs core-shell nanofibers. They used the phase change thermochromic materials (PCTMs) to make an improvement to PCMs. Displaying in Figure 14, PCTM system of crystal violet lactone (CVL) as dye and bisphenol $\mathrm{A}$ as developer mix in fatty alcohol or fatty acid was chosen (Burkinshaw, et al., 1998; Hirata, et al., 2006; MacLaren, et al., 2005). As a traditional PCTM, it has a simple component and stable thermochromic property, and the thermochromic temperature can be adjusted by changing fatty alcohol or fatty acid filling. (Here, 1-Tetradecanol 37 $39^{\circ} \mathrm{C}$ PCM was chosen for the good prospect in intelligent senors and devices of body temperature materials.) And CVL, bisphenol A and 1-tetradecanol (the mixture system was abbreviated to $\mathrm{CBT}$ ) were chosen as the inner loading material for the melt coaxial electrospinng.
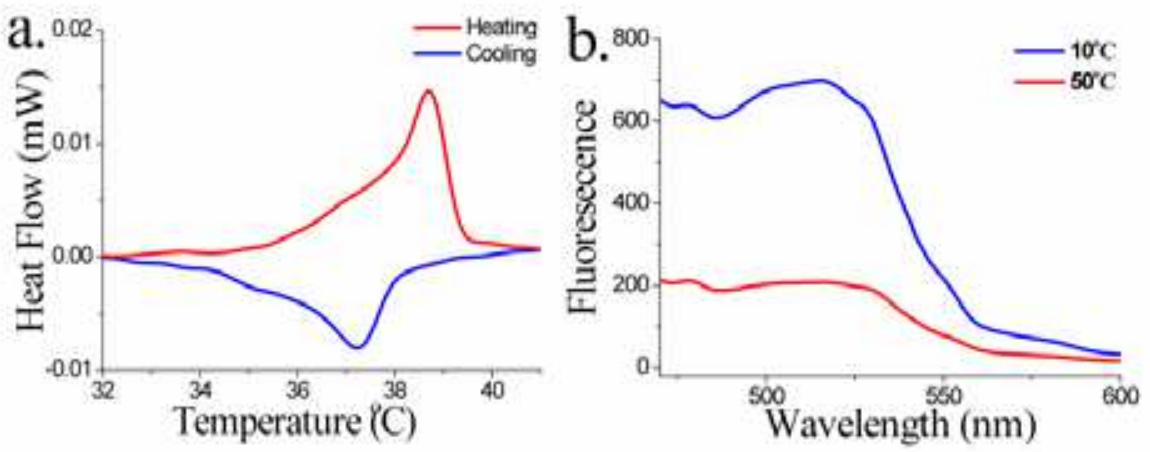

Fig. 15. a) DSC measurements cycle curve and b) Fluorescence spectra of the CBT-PMMA nanofibers at $10^{\circ} \mathrm{C}$ and at $50^{\circ} \mathrm{C}$.

After successful encapsulation by melt coaxial electrospinng, (Figure 11) these CBT-core and PMMA-shell nanofiber non-woven materials show some excellent properties. Figure 15 indicates the thermo-responsive property. Differential scanning calorimetry (DSC) experiment gave the DSC cycle curve of CBT-PMMA fibers, which revealed an obvious absorption and release process in the DSC measurement cycle. (Figure 15a) The acuate peak and vale of DSC cycle curve indicates that the CBT-PMMA core-shell nanofibers have a more sensitive phase-transformation behaviour than a bulk CBT mixture. (McCann et al., 2006; Li et al, 2009) Figure 15b shows the fluorescence spectra below and beyond the phase change temperature. At the freezing state of $\mathrm{CBT}$ at $10^{\circ} \mathrm{C}$, the fibers have strong fluorescent emission at $503 \mathrm{~nm}$, which is the characteristic fluorescence of CVL in CBT system. With temperature increased to $50{ }^{\circ} \mathrm{C}$, the emission intensity of the fibers has a great decrease. When temperature is decreased to $10^{\circ} \mathrm{C}$, the emission intensity was reverted again. The fluorescent change of the fibers in the heating-cooling process showed the obvious thermochromism.

PMMA polymer was used as the out shell material to encapsulate the thermochromic material, due to its good optical transparent property, which always be used for organic glass. Figure 16 displays the fluorescent signal and image under fluorescence microscope. With UV light exciting, the CBT-PMMA nanofibers showed good green emission and got 
clear fluorescence image. That makes the encapsulation of fluorescence thermo-chromic materials in core-shell nanofibers have an insight into the thermo-responsive senor.

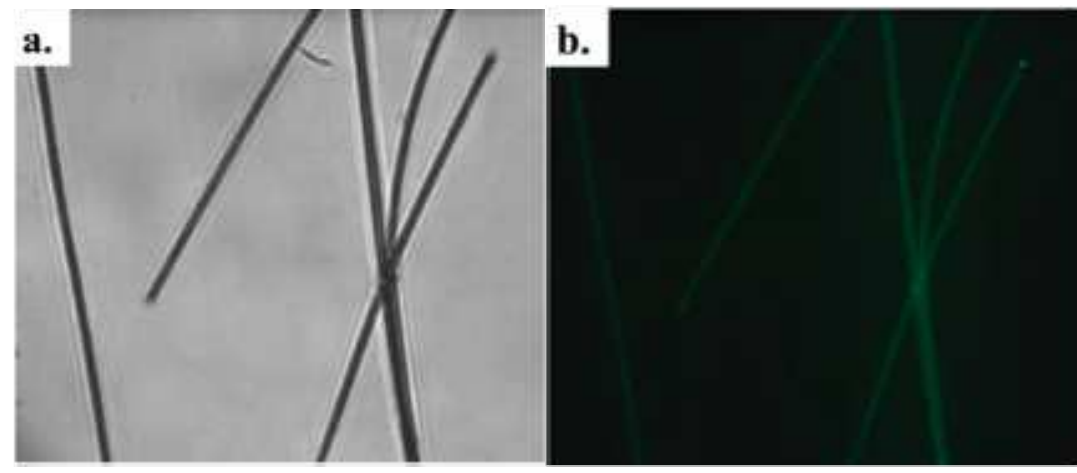

Fig. 16. (a) Optical and (b) fluorescent images of CBT-PMMA nanofibers of fluorescence microscope.

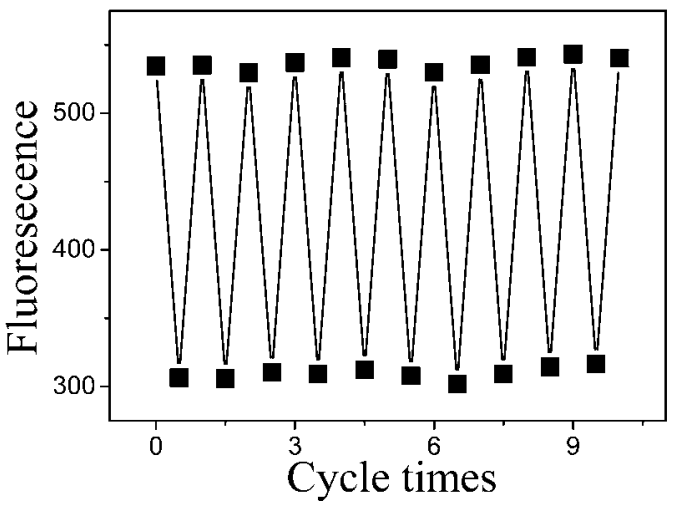

Fig. 17. The thermochromic cycle reversibility experiment of CBT-PMMA nanofibers' film. In each cycle, the fluoresecent emission at $\lambda=503 \mathrm{~nm}$ of the samples was monitored at $10^{\circ} \mathrm{C}$ and $50^{\circ} \mathrm{C}$, respectively.

The thermochromic reversibility cycle experiments of the CBT-PMMA fibers film were investigated to check the responsive stability. The fluidity after melting is the main limiting factor for the PCTMs practical application. The encapsulation of PCTMs in micro/nano matrix to stabilize the PCTMs could solve the problem. In Figure 17, ten heating-cooling cycles between $10-50{ }^{\circ} \mathrm{C}$ were performed, and the fluorescent maximal emission at $503 \mathrm{~nm}$ of the CBT-PMMA fibers film was monitored. There was not any essential loss in fluorescent characteristics during the repeated thermochromism processes. It proved that the CBTPMMA core-shell nanofibers showed good fluorescence thermochromic reversibility. The encapsulation of CBT in PMMA nanofibers realizes the device and practical application of PCTMs CBT. It has new insight into the preparation of temperature sensors with good 
fluorescence signal, and body temperature calefactive materials with intelligent thermal energy absorbing, retaining and releasing.

Melt coaxial electrospinning is one good example to fabricate functional core-shell nanofiber materials. By introducing different responsive or functional materials as the core and choosing adaptable polymer, we could accomplish the novel functionality and function modification. Thus we could perform versatile modification and control to realize multifunction and diversification, for the multi-channel coaxial electrospinning example. As the extension and development of coaxial electrospinning, melt coaxial electrospinning shows good application performance and controllability. It indicates the generality of electrospinning for one-dimensional nanomaterials fabrication.

\subsubsection{Future of core-shell nanofibers: Multi-encapsulation and Multi-responsive materials.}

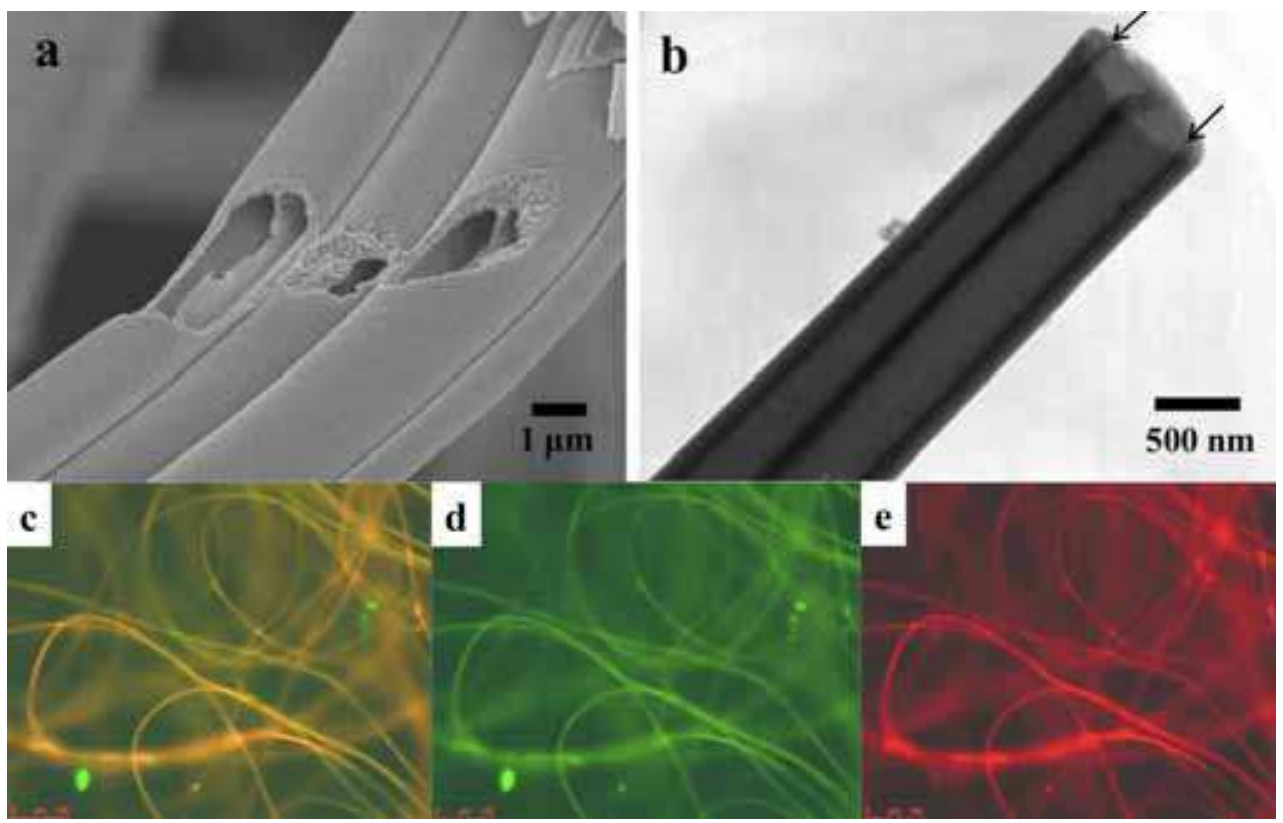

Fig. 18. SEM (a) and TEM (b) image of multichannel nanofibers, which could be loaded with different fluorescence materials, and colourful fluorescence images (c)-(e) of multiencapsulation core-shell nanofibers.

Diversification and integration is the pilot of the science research on philosophy. And for the aspects and prospects of the new materials developing currently and future, the multifunctional, integrative and miniature devices researches are greatly and urgently expected. At the same time for core-shell nanofibers, diversification and multifunction will be the main aspects in the future. Coaxial electrospinning provides the flexible and facilitate method to construct and fabricate diversiform nano- or micro- encapsulation materials and core-shell nano- or micro- devices. Figure 18 shows some primary research of the multi- 
encapsulation and multi-responsive materials in the core-shell nanofibers by coaxial electrospinning. In the cavums centre of the fibers, rhodamine $\mathrm{B}$ and fluorescein isothiocyanate (FITC) et al. dyes were used as the core loading materials to make the multichannel stain. Then, colourful fluorescence images of multi-loading integrative core-shell nanofibers were obtained as shown in Figure 18 (c)-(e). We could believe that: with more versatile responsive materials loading or encapsulation, one can obtain the more multifunction nanofibers materials by coaxial electrospinning.

\section{Conclusion}

Compared with self-assembly of molecular building blocks or template printing et al. methods, coaxial electrospinning can be used to prepare various organic or inorganic tubular nanostructures fast and facilely. With better controlling, coaxial electrospinning can realize diversification and encapsulation of nanofibers with tubular or core-shell second nanostructures. Multichannel nanotubes have ultra-large specific surface area, isolation nanostructure and continuous nanotube. In core-shell nanofibers, as core, varied responsive materials were independently encapsulated into polymer-shell. The materials were fixed and protected, but the responsive properties were kept. With nano-space encapsulating and ultra-large specific surface area, the responsive core-shell nanofibers materials are more sensitive on stimuli-responsive properties. These core-shell nanofibers or nanotubes have great applications in catalysis, fluidics, ptrification, separation, gas storage, energy conversion and storage, drug release, sensing, and environmental protection. Creating and accurate controlling 1D nanomaterials with multicompartmental inner structures is still a great challenge. It is believed that the core-shell nanofibers will give a wide space to scientists to show more creativity at the nano-channel and nano-encapsulation domain.

\section{Acknowledgments}

The authors thank the Natural Science Foundation of China (NSFC), the Ministry of Science and Technology (MOST) of China, for continuous financial support.

The authors are grateful to Prof. Lei Jiang for great help on technology and knowledge. They are also grateful to the Chinese Academy of Sciences.

\section{References}

Adamson, A. W., Gast, A. P. (1997). Physical Chemistry of Surface, Sixth Edition, 1997, John Wiley \& Sons, Inc.

Burkinshaw, S. M.; Griffiths, J.; Towns, A. D. (1998). Reversibly thermochromic systems based on pH-sensitive functional dyes. el Mater. Chem., Vol. 8, 1998, pp. 2677-2683.

Doshi, J.; Reneker, D. H. (1995). Electrospinning process and applications of electrospun fibers, el Electrostat., Vol. 35, 1995, pp. 151-160.

Exerowa, D., Kruglyakov, P. M. (1998). Foam and foam films: Theory, experiment, application. 1998, Elsevier.

Gil, E. S.; Hudson, S. M. (2004). Stimuli-reponsive polymers and their bioconjugates, Prog. Polym. Sci., Vol. 29, 2004, pp. 1173-1222. 
Greiner, A.; Wendorff, J. H. (2007). Electrospinning: A fascinating method for the Preparation of Ultrathin Fibers, Angew. Chem. Int. Ed., Vol. 46, 2007, pp 5670-5703.

Hirata, S.; Watanabe, T. (2006). Reversible thermoresponsive recording of fluorescent images (TRF), Adv. Mater., Vol. 18, 2006, pp. 2725-2729.

Höhler, R., Cohen-Addad, S. (2005). Rheology of liquid foam, el Phys.: Condens. Matter. Vol. 17, 2005, pp. 1041-1069.

Hu, J. T., Odom, T. W., Lieber, C. M. (1999). Chemistry and physics in one dimension: synthesis and properties of nanowires and nanotubes, Acc. Chem. Res., Vol. 32, 1999, pp. 435-445.

Huang, Z.-M.; Zhang, Y.-Z.; Kotaki, M.; Ramakrishna, S. (2003). A review on polymer nanofibers by electrospinning and their applications in nanocomposites, Compos. Sci. Technol., Vol. 63, 2003, pp. 2223-2253.

Law, M., Goldberger, J., Yang, P. D. (2004). Semiconductor nanowires and nanotubes, Ann. Rev. Mater. Res., Vol. 34, 2004, pp. 83-122.

Li, D.; Xia, Y. (2004). Electrospinning of nanofibers: Reinventing the wheel? Adv. Mater., Vol. 16, 2004, pp 1151-1170.

Li, D.; Xia, Y. (2004). Direct fabrication of composite and ceramic hollow nanofibers by electrospinning, Nano Letters, Vol. 4, 2004, pp 933-938.

Li, F.; Zhao, Y.; Wang, S.; Han, D.; Jiang, L.; Song, Y. (2009). Thermochromic core-shell nanofibers fabricated by melt coaxial electrospinning, el Appl. Polymer Sci., Vol. 112, 2009, pp 269-274.

Loscertales, I. G., Barrero, A., Guerrero, I., Cortijo, R., Marquez, M., Ganan-Calvo, A. M. (2002). Micro/nano encapsulation via electrified coaxial liquid jets, Science, Vol. 295, 2002, pp.1695-1698.

Lu, Y.; Liu, J. (2007). Smart nanomaterials inspired by biology: Dynamic assembly of errorfree nanomaterials in response to multiple chemical and biological stimuli, Acc. Chem. Res., Vol. 40, 2007, pp. 315-323.

MacLaren, D. C.; White, M. A. (2005). Design rules for reversible thermochromic mixtures, $d$ Mater. Sci., Vol. 40, 2005, pp. 669-676.

McCann, J. T.; Marquez, M.; Xia, Y. (2006). Melt coaxial electrospinning: A versatile method for the encapsulation of solid materials and fabrication of phase change nanofibers, Nano Letters, Vol. 6, 2006, pp. 2868-2872.

Mulligan, J. C.; Colvin, D. P.; Bryant, Y. G. (1996). Microencapsulated phase-change material suspensions for heat transfer in spacecraft thermal systems, el Spacecraft Rockets, Vol. 33, 1996, pp. 278-284.

Reneker, D. H.; Chun, I. (1996). Nanometre diameter fibres of polymer, produced by electrospinning, Nanotechnology, Vol. 7, 1996, pp. 216-223.

Sun, Z. C., Zussman, E., Yarin, A. L., Wendorff, J. H., Greiner, A. (2003). Compound coreshell polymer nanofibers by co-electrospinning, Adv. Mater., Vol. 15, 2003, 19291932.

Zalba, B.; Marín, J. M.; Cabeza, L. F.; Mehling, H. (2003). Review on thermal energy storage with phase change: materials, heat transfer analysis and applications, Appl. Thermal. Eng., Vol. 23, 2003, pp. 251-283. 
Zhang, Y.; Huang, Z-M.; Xu, X.; Lim, C. T.; Ramakrishna, S. (2004). Preparation of core-shell structured PCL-r-Gelatin bi-Component nanofibers by coaxial electrospinning, Chem. Mater., Vol. 16, 2004, pp 3406-3409.

Zhao, Y.; Cao, X.; Jiang, L. (2007). Bio-mimic multichannel microtubes by a facile method, $d$ Am. Chem. Soc., Vol. 129, 2007, pp 764-765. 


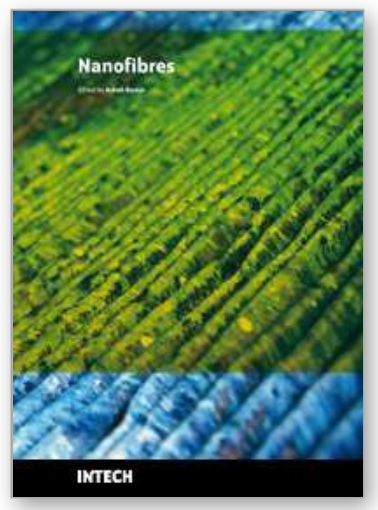

\author{
Nanofibers \\ Edited by Ashok Kumar
}

ISBN 978-953-7619-86-2

Hard cover, 438 pages

Publisher InTech

Published online 01, February, 2010

Published in print edition February, 2010

"There's Plenty of Room at the Bottom" this was the title of the lecture Prof. Richard Feynman delivered at California Institute of Technology on December 29, 1959 at the American Physical Society meeting. He considered the possibility to manipulate matter on an atomic scale. Indeed, the design and controllable synthesis of nanomaterials have attracted much attention because of their distinctive geometries and novel physical and chemical properties. For the last two decades nano-scaled materials in the form of nanofibers, nanoparticles, nanotubes, nanoclays, nanorods, nanodisks, nanoribbons, nanowhiskers etc. have been investigated with increased interest due to their enormous advantages, such as large surface area and active surface sites. Among all nanostructures, nanofibers have attracted tremendous interest in nanotechnology and biomedical engineering owing to the ease of controllable production processes, low pore size and superior mechanical properties for a range of applications in diverse areas such as catalysis, sensors, medicine, pharmacy, drug delivery, tissue engineering, filtration, textile, adhesive, aerospace, capacitors, transistors, battery separators, energy storage, fuel cells, information technology, photonic structures and flat panel displays, just to mention a few. Nanofibers are continuous filaments of generally less than about $1000 \mathrm{~nm}$ diameters. Nanofibers of a variety of cellulose and non-cellulose based materials can be produced by a variety of techniques such as phase separation, self assembly, drawing, melt fibrillation, template synthesis, electrospinning, and solution spinning. They reduce the handling problems mostly associated with the nanoparticles. Nanoparticles can agglomerate and form clusters, whereas nanofibers form a mesh that stays intact even after regeneration. The present book is a result of contributions of experts from international scientific community working in different areas and types of nanofibers. The book thoroughly covers latest topics on different varieties of nanofibers. It provides an up-to-date insightful coverage to the synthesis, characterization, functional properties and potential device applications of nanofibers in specialized areas. We hope that this book will prove to be timely and thought provoking and will serve as a valuable reference for researchers working in different areas of nanofibers. Special thanks goes to the authors for their valuable contributions.

\title{
How to reference
}

In order to correctly reference this scholarly work, feel free to copy and paste the following:

Fengyu Li, Yong Zhao and Yanlin Song (2010). Core-Shell Nanofibers: Nano Channel and Capsule by Coaxial Electrospinning, Nanofibers, Ashok Kumar (Ed.), ISBN: 978-953-7619-86-2, InTech, Available from: http://www.intechopen.com/books/nanofibers/core-shell-nanofibers-nano-channel-and-capsule-by-coaxialelectrospinning 
open science | open minds

InTech Europe

University Campus STeP Ri

Slavka Krautzeka 83/A

51000 Rijeka, Croatia

Phone: +385 (51) 770447

Fax: +385 (51) 686166

www.intechopen.com
InTech China

Unit 405, Office Block, Hotel Equatorial Shanghai

No.65, Yan An Road (West), Shanghai, 200040, China

中国上海市延安西路65号上海国际贵都大饭店办公楼 405 单元

Phone: +86-21-62489820

Fax: +86-21-62489821 
(C) 2010 The Author(s). Licensee IntechOpen. This chapter is distributed under the terms of the Creative Commons Attribution-NonCommercialShareAlike-3.0 License, which permits use, distribution and reproduction for non-commercial purposes, provided the original is properly cited and derivative works building on this content are distributed under the same license. 